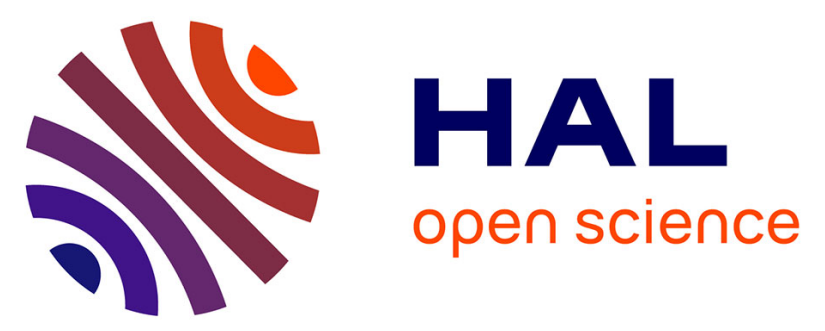

\title{
La fouille du trésor monétaire de Saint-Germain-de-Varreville (Manche) : stratigraphie d'un pécule de 14528 nummi (première moitié du IVe siècle)
}

\author{
Pierre-Marie Guihard, Cécile Allinne, Eric Broine
}

\section{To cite this version:}

Pierre-Marie Guihard, Cécile Allinne, Eric Broine. La fouille du trésor monétaire de Saint-Germainde-Varreville (Manche) : stratigraphie d'un pécule de 14528 nummi (première moitié du IVe siècle). Annales de Normandie, 2013, pp.3-25. 10.3917/annor.631.0003 . hal-02025358

\section{HAL Id: hal-02025358 https://hal.science/hal-02025358}

Submitted on 19 Feb 2019

HAL is a multi-disciplinary open access archive for the deposit and dissemination of scientific research documents, whether they are published or not. The documents may come from teaching and research institutions in France or abroad, or from public or private research centers.
L'archive ouverte pluridisciplinaire HAL, est destinée au dépôt et à la diffusion de documents scientifiques de niveau recherche, publiés ou non, émanant des établissements d'enseignement et de recherche français ou étrangers, des laboratoires publics ou privés. 


\title{
La fouille du trésor monétaire de Saint-Germain-de-Varreville (Manche) : stratigraphie d'un pécule de 14528 nummi (première moitié du Iv siècle)
}

\author{
Pierre-Marie GUIHARD* et CécIle ALlinNE**, \\ avec la collaboration d'ÉRIC BROINE***
}

$\mathrm{E}$

N 2011, sur un terrain légèrement en pente qui domine le lit d'un petit cours d'eau, le " ruisseau de By ", à quelques centaines de mètres au sud du village de Saint-Germain-de-Varreville (Manche), des travaux agricoles ont mis au jour un trésor de monnaies romaines de la première moitié du IV siècle (Fig. 1). Conservé dans son contenant d'origine, un gros vase en céramique commune, il constitue une découverte tout à fait exceptionnelle. Grâce au civisme de l'inventeur et à la collaboration entre le Centre Michel de Boüard-CRAHAM et le Service régional de l'Archéologie de Basse-Normandie, nous disposons aujourd'hui d'un ensemble dont l'intégralité a pu être préservée, situation qui, si naturelle qu'elle puisse sembler, n'en demeure pas moins un cas de figure trop peu fréquent méritant d'être souligné ${ }^{1}$.

Le trésor avait été découvert dans un sol limoneux entre 40 et $60 \mathrm{~cm}$ de profondeur. Son aspect extérieur surprenait par son état de conservation général. Contenu dans un vase de forme ovoïde en terre noire, d'une trentaine de centimètres de hauteur, il semblait comme avoir été figé par le temps : aucune brisure et un remplissage intact, inviolé depuis des siècles. Mais le plus spectaculaire se trouvait à l'ouverture du vase : un millefeuille de monnaies plus ou moins concrétionnées, qui ne laissait pas la moindre place à des espaces vides. Afin de vérifier s'il en était de même à l'intérieur du vase, une radiographie a

\footnotetext{
* $\quad$ Service de numismatique, Centre Michel de Boüard, CRAHAM, UMR 6273 (CNRS / Université de Caen Basse-Normandie).

** Maître de conférences, Centre Michel de Boüard, CRAHAM, UMR 6273 (CNRS / Université de Caen Basse-Normandie).

**** Archéomaticien, Centre Michel de Boüard, CRAHAM, UMR 6273 (CNRS / Université de Caen Basse-Normandie).

1 Nous tenons à adresser nos remerciements à M. Fichet de Clairefontaine (Conservateur en chef, Service régional de l'Archéologie) pour avoir confié l'étude du trésor au service de numismatique du Centre Michel de Boüard-CRAHAM.
} 


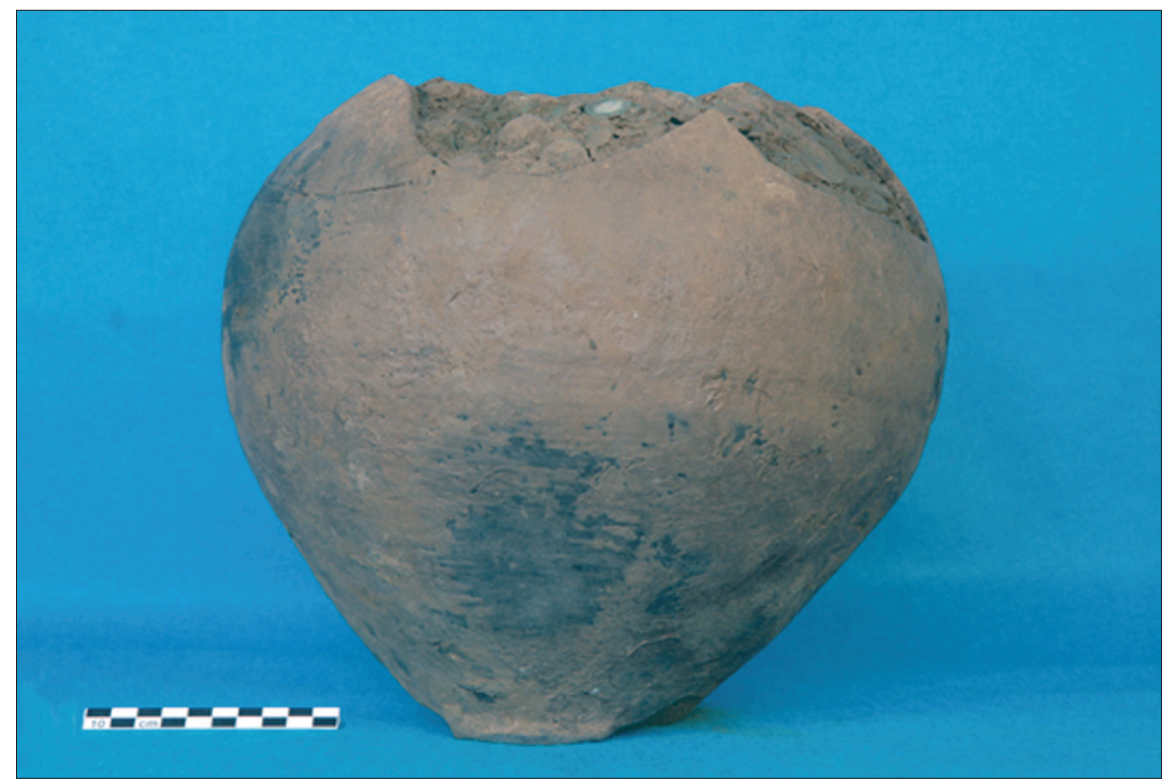

Fig. 1 (Crédits photographiques, Éric Broine / CRAHAM 2011).

été effectuée. Elle a révélé que le volume global était bien occupé par un agrégat très dense de monnaies. Le tout pesait $42 \mathrm{~kg}$, dont nous avons retiré le poids de la céramique (estimé à $3,8 \mathrm{~kg}$ ). La masse monétaire concrétionnée fut ainsi évaluée à $38,2 \mathrm{~kg}$.

L'enfouissement de Saint-Germain-de-Varreville correspond à une forme de sécurisation qui trouve de multiples parallèles tout au long de l'histoire monétaire des civilisations. Comme le fait remarquer l'historien grec Xénophon à propos de l'argent : " on n'en a jamais assez pour n'en plus désirer, et, si l'on possède une grande quantité, on ne prend pas moins de plaisir à enfouir son surplus qu'à en faire usage »(Les Revenus, IV, 7)2. Depuis que la numismatique existe, les trésors monétaires ont toujours constitué un enjeu important pour dater et comprendre l'organisation des frappes, mais, jusqu'à présent, le mode de remplissage des contenants de trésors a été peu étudié. Comment étaient rangées les monnaies dans les conteneurs? Étaient-elles disposées en vrac ou soigneusement organisées, en sacs ou en rouleaux par exemple ? Peut-on identifier des étapes dans le processus de thésaurisation, c'est-à-dire des phases successives de remplissage ? Sur quelle base alors distinguer les limites entre les différents lots, notamment si les tissus, cuirs, ou autres emballages ont disparu? Ces questions ont constitué, en juillet 2012, le cœur d'un projet de micro-fouille au service de

2 Xénophon, Les revenus. Texte établi, traduit et commenté par E. Talbot, Eurres complètes de Xénophon, t. I, Paris, Hachette, 1859. 
numismatique du Centre Michel de Boüard-CRAHAM (UMR 6273, Université de Caen Basse-Normandie), destiné à étudier l'agencement des monnaies dans le trésor de Saint-Germain-de-Varreville. Si notre article fait ainsi une large place aux questions de méthode, sa finalité dépasse largement ce cadre. L'objectif est d'essayer de révéler des pratiques de thésaurisation, susceptibles de constituer des indices pour la compréhension des comportements de l'usager face à son pécule, c'est-à-dire des modes de gestion de sa ressource monétaire.

\section{DÉPÔT MONÉTAIRE ET FOUILLE EN LABORATOIRE : QUELLE MÉTHODE ?}

Au début des années 1980, les Britanniques Besly et Bland ont été les premiers à plaider pour une approche archéologique des trésors monétaires. En analysant le contenu des dépôts d'Aldbourne (Wiltshire) et de Tattershall (Lincolnshire), ils ont pu montrer que les monnaies avaient été placées avec soin à l'intérieur des récipients ${ }^{3}$. Cette approche allait permettre à d'autres recherches de se développer ${ }^{4}$. Ainsi, au fil des ans, des numismates français, allemands et luxembourgeois se sont intéressés au mode de remplissage des trésors ${ }^{5}$. Petit à petit, une ébauche méthodologique s'est dessinée, qui, en terme de stratégie, devait beaucoup au protocole opératoire appliqué à la fouille d'urnes funéraires. Les résultats obtenus ont apporté, dans certains cas, des informations très strictes sur le mode de remplissage des dépôts. Ils ont en particulier montré que l'intérieur des récipients était stratigraphié, et pouvait être organisé au moyen de sacs ou de rouleaux de monnaies.

Ce succès a encouragé le développement de projets plus ambitieux. C'est le cas de l'étude du trésor de Pannecé (Loire-Atlantique), qui marque un véritable tournant dans la mise en place d'une méthodologie adaptée à la fouille

3 E. Besly, "The Aldbourne, Wilts., Hoard ", Coin Hoards from Roman Britain, IV, 1984, p. 63-104 ; E. Besly, R. Bland, "The Tattershall Thorp, Lincs., Hoard ", Coin Hoards from Roman Britain, IV, 1984, p. 105-138.

4 Quelques rares expériences très imparfaites les ont néanmoins précédées. Il s'agit notamment du trésor découvert en 1895 à Cologne (Rheinland-Pfalz), qui est à notre connaissance la première étude qui prête attention au contenu d'un dépôt monétaire. En effet, les numismates qui ont étudié ce trésor, enfoui vers 333, ont remarqué que les monnaies étaient rangées en rouleaux de 50 : V. BAHRFELD, Münzen Constantinus d. Gr. und seiner Zeit aus dem Münzfunde von Köln a. R., 1895, Halle, A. Riechmann, 1923. Voir aussi les observations faites sur le trésor d'Horath : H. Cuppers, «Trésor d'Horath, 1964 (canton de Bernkastel) ", Archäologische Funde im Landkreis Bernkastel, Zeltigen, Bernkastel-Kues, Lankreis Bernkastel, 1966, p. 99 et pl. 51.

5 M. Amandry et alii, «Le trésor d'antoniniens des Communaux d'Arésieux à Villette-d'Anthon (Isère) ", Bulletin de la Société française de Numismatique, juin 1996, p. 117-123 ; S. Estiot, "Le trésor de Saint-Maurice-de-Gourdans (Ain) : une tirelire du III siècle de notre ère ", Bulletin de la Société française de Numismatique, juin 1996, p. 124-127; S. Esт гот, «Le trésor de Saint-Maurice-de-Gourdans - Pollet (Ain), 1272 antoniniens stratigraphiés", Trésors Monétaires, XVI, 1997, p. 69-127 ; H.-M. Von KaENEL et alii, Der Münzhort aus dem Gutshof in Neftenbach: Antoniniane und Denare von Septimius Severus bis Postumus, Zurich, Fotorotar AG, 1993 (Zürcher Denkmalpflege. Archäologische Monographien, 16) ; R. Weiller, Der Schatzfund vom Titelberg (1995) : Antoniniane von Caracalla bis Aurelian, Berlin, G. Mann, 1999 (Studien zu Fundmüzen der Antike, 15). 
de trésor ${ }^{6}$. Pour la première fois, l'enquête se déroule en laboratoire, à l'aide de moyens techniques importants pour garantir un enregistrement précis et continu des données. Le contenu du dépôt se voit donc attribuer une attention qu'aucune initiative jusqu'alors n'avait mis en ouvre pour recueillir des informations sur la constitution d'un trésor monétaire. Les résultats sont à la hauteur des travaux engagées ${ }^{7}$. Ceux-ci ont en effet permis d'identifier des déposes successives d'ensembles monétaires, qui étaient probablement à l'origine contenus dans des «sacs». Les poids des monnaies qui composent ces lots ont été également étudiés. Après avoir été convertis en livres romaines, tous sont très proches de nombres entiers (quatre, cinq ou huit livres), ce qui pourrait indiquer que les sacs ont été constitués par la pesée des monnaies. L'étude a en outre permis de déterminer avec précision la position stratigraphique de chaque ensemble, dont l'agencement avait été, semble-t-il, organisé avec logique et en vue d'optimiser le remplissage du vase, afin de placer un maximum de lots dans le volume disponible. C'est donc l'approche stratigraphique du remplissage qui a contribué le plus efficacement à mieux connaître le dépôt.

La fouille des lots et couches de monnaies repérées dans le vase de Pannecé prolonge ainsi une méthode inaugurée par les Britanniques pour appréhender la constitution d'un dépôt monétaire. Mais dans cette direction elle va beaucoup plus loin, puisqu'elle ouvre sur une exploration approfondie du mode de thésaurisation. Surtout, elle conforte l'idée que le contenu d'un trésor doit être considéré comme une structure archéologique à part entière et traité avec les méthodes de la discipline. Cette évidence a précisément guidé notre étude du trésor de Saint-Germain-de-Varreville.

\section{Moyens mis en ceuvre : l'enregistrement des données}

Un trésor composé de plusieurs dizaines de kilogrammes de monnaies concrétionnées constitue une structure archéologique complexe. La principale difficulté réside dans la bonne compréhension de sa stratigraphie. La fouille s'opère sur un espace réduit et l'intérieur du dépôt n'est accessible qu'en détruisant à la fois, progressivement, la poterie et les couches de monnaies les unes après les autres. Comme dans toute fouille archéologique, il est impossible de revenir en arrière pour affiner une observation, et tous les gestes effectués par les fouilleurs doivent alors être minutieusement et méthodiquement enregistrés, sous la forme de descriptions écrites, codifiées et de documents graphiques et photographiques. L'ensemble de ces actes forme les archives de la fouille. Celles-ci transcrivent le plus objectivement possible tout ce qui a été fait, vu et

6 G. Aubin (dir.), «Dossier : le trésor monétaire de Pannecé II (Loire-Atlantique, France) », Bulletin de la Société française de Numismatique, février 2005, p. 26-42.

7 Voir l'article de C. CÉcillon, "La micro-fouille du vase B du trésor de Pannecé II (LoireAtlantique) : mode opératoire et résultats ", dans G. AuBin (dir.), op. cit., 2005, p. 32-41. 
compris, et représentent tout ce qu'il reste du terrain une fois la fouille achevée. Ultime témoignage, elles doivent permettre de reconstituer fidèlement l'objet d'étude depuis le moment où il était intact jusqu'à la fin de son démontage.

Pour permettre d'analyser ce terrain d'investigation peu banal et de voyager au cœur du trésor de Saint-Germain-de-Varreville, divers moyens techniques ont été mis en œuvre, garantissant le bon déroulement de la fouille et la fiabilité des enregistrements effectués ${ }^{8}$. Nous avons d'abord stabilisé le vase, dans une position qui ne devait plus pouvoir varier le temps de l'opération. Le pot a ainsi été logé dans un coffrage de maintien en mousse haute densité et installé sur une table fixe, de sorte à dominer l'ouverture du récipient au moment du démontage. De façon complémentaire, une grande armature métallique a été montée, à la manière d'une cage, autour de la table d'opération. Spécialement conçue à cette occasion, elle a servi à l'installation du matériel photo et vidéo, ainsi qu'à la fixation des systèmes d'éclairage (Fig. 2). Ces dispositifs ont notamment servi à réaliser des prises de vue depuis des angles fixes, rendant compte progressivement de l'enlèvement des couches de monnaies. L'immobilisation du pot nous a aussi permis de quadriller la surface de fouille en quatre secteurs, dans lesquels ont été localisés et référencés les différents ensembles monétaires mis en évidence, et de favoriser des prises de mesures altimétriques.

Le système d'enregistrement des données de fouille que nous avons élaboré prend modèle sur ce qui est appliqué en archéologie de terrain. L'objectif est d'établir la mémoire de la fouille et préciser le plus finement possible les caractéristiques du dépôt et ses éléments constitutifs. Pour cela, nous avons conçu une fiche descriptive pour chaque ensemble de monnaies identifié ${ }^{9}$ (ou fiche d'unité stratigraphique). Elle est composée de trois grandes rubriques. La première vise à restituer la position du lot dans le pot et à en décrire sa morphologie générale, en trois dimensions. Ces informations, données sous forme écrite complétée de schémas et dessins, sont aussi reportées sur des tirages photographiques, sur lesquels figurent les contours et surtout les cotes altimétriques des groupes de monnaies. Une deuxième rubrique regroupe la description de la structure interne du dépôt, c'est-à-dire des monnaies, bien sûr, mais aussi des sédiments pris entre les pièces ou d'autres objets éventuels (vestiges de cuir, de bois, de tissus, ou encore débris végétaux ou animaux).

8 Ces derniers ont déjà fait l'objet d'une rapide présentation, P.-M. Guihard, C. Allinne et É. Broine, "Fouille inédite autour d'un trésor romain ", Connexions, 50, septembre 2012, p. 4.

9 Nous avons en fait distingué deux types d'unités stratigraphiques : les « faits », correspondant aux lots monétaires cohérents possédant des limites sûres et bien définies, et les " passes ", correspondant à des groupes de monnaies prélevées dans des ensembles dont les limites n'étaient pas nettement visibles. Cette dernière catégorie d'unité stratigraphique a été utilisée lors de la fouille de lots comprenant un nombre très important de pièces (plus de 3000 ), afin de limiter le risque de mélange de spécimens provenant de groupes différents, au cas où nous n'aurions pas réussi à identifier correctement leurs limites. Au total, toutes ces «passes » ont pu être rattachées à des «faits ». La structure des fiches d'enregistrement de « fait » et de « passe » était la même. 


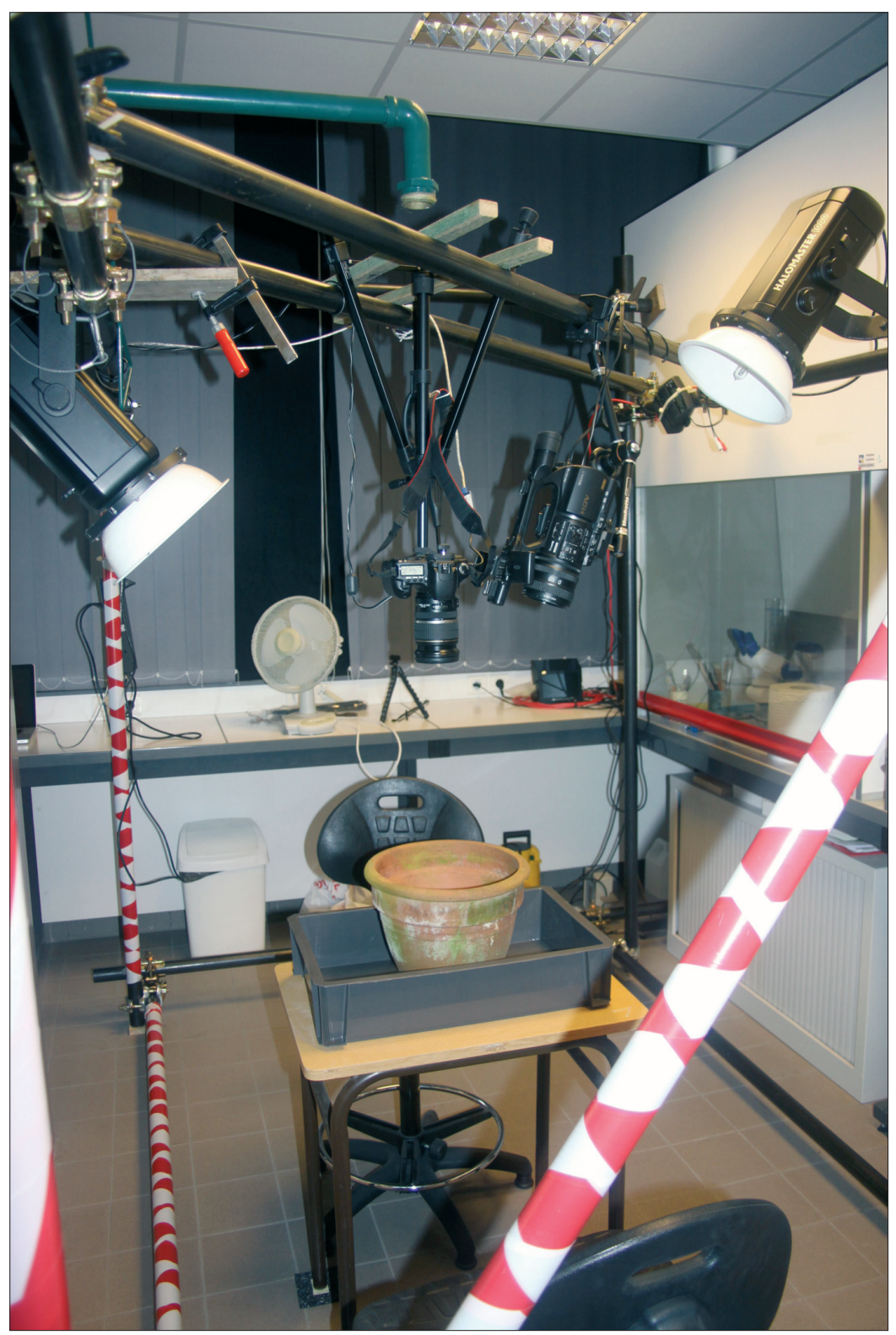

Fig. 2.

Une attention particulière a été portée à la définition de l'inclinaison des pièces et de leur agencement, ainsi qu'au type de corrosion qui les affectait, et à la nature du fin sédiment qui les recouvrait. Ces différents caractères constituent en effet des indices pour isoler les lots monétaires et différencier des phases 
de déposition. Enfin, une troisième rubrique était consacrée aux propositions d'interprétation effectuées à partir de ces premières observations.

Parallèlement, nous avons décidé de prendre appui sur les nouvelles technologies. Grâce à elles, il sera possible de pénétrer virtuellement et de façon pédagogique dans les coulisses de l'étude scientifique d'une découverte rare. La fouille s'est ainsi déroulée sous l'objectif d'une caméra. Pour cela, nous avons fait appel au département ressources audiovisuelles et multimédias du $\mathrm{CRISI}^{10}$, dirigé par S. Gorzkowski. Nous nous sommes également rapprochés de l'équipe Image du GREYC ${ }^{11}$ pour produire un document 3D qui accompagnera la vidéo, prochainement disponible sur Internet.

\section{Voyage au centre du trésor}

Reconnaître les différentes composantes du trésor et en restituer la stratigraphie n'ont pas été pour autant des étapes faciles. Cet exercice, qui tient du déshabillage, impose de repérer et de libérer dans la masse feuilletée de monnaies les éventuels ensembles atypiques ou homogènes. Ce que nous cherchions, c'était la manière dont le thésaurisateur avait constitué et utilisé son pécule.

Les perspectives ouvertes par ce projet ont nécessité de faire le choix d'un mode opératoire défini. En s'appuyant sur les méthodes de l'archéologie dite sédimentaire, nous avons procédé à une fouille que l'on pourrait qualifier d' " extensive » : les monnaies ont été dégagées depuis la surface du vase vers l'intérieur du dépôt, par couches horizontales, et du lot le plus récent au lot le plus ancien (Fig. 3). Le but était d'éviter de créer des cavités qui auraient nui à la lecture spatiale du remplissage et de respecter l'ordre de déposition des ensembles, en ôtant les couches une par une comme si l'on remontait le temps. Toutes les monnaies prélevées ont été au fur et à mesure comptées et enregistrées, puis placées dans des sacs hermétiques référencés par lot, nommés "faits ", ou "passes ». Parallèlement, les sédiments et les résidus organiques piégés au milieu des monnaies ont été prélevés pour être examinés et identifiés sous loupe binoculaire.

Dans cette approche du contenu, la position des monnaies, principal indicateur des modes d'agencement du dépôt, a été déterminante. Nous l'avons étudiée à travers les variations d'inclinaison. Les observations effectuées au cours de la fouille ont vite révélé deux principales configurations. Ainsi, nous avons constaté que des monnaies étalées à plat sur toute la surface disponible

10 Centre de Ressources Informatiques et de Services d'Information de l'Université de Caen BasseNormandie

11 Groupe de Recherche en Informatique, Image, Automatique et Instrumentation de Caen (UMR 6072) (https://www.greyc.fr). 


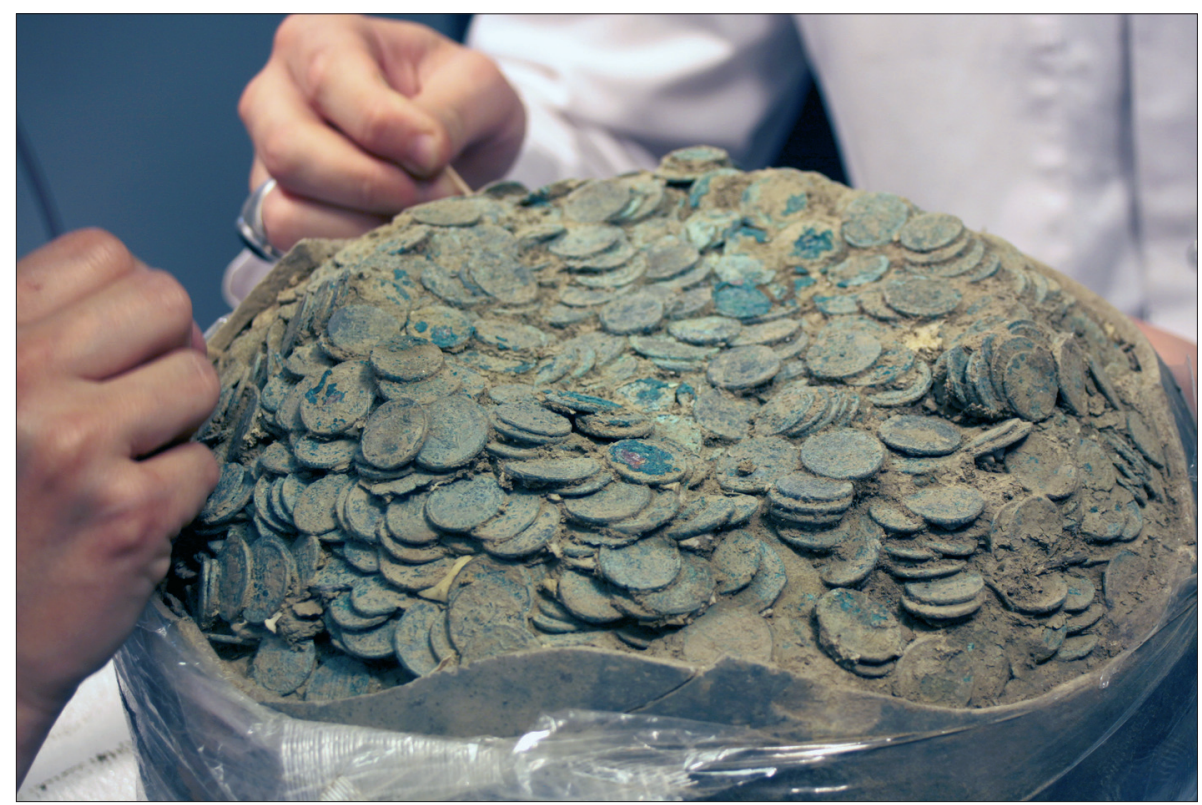

Fig. 3.

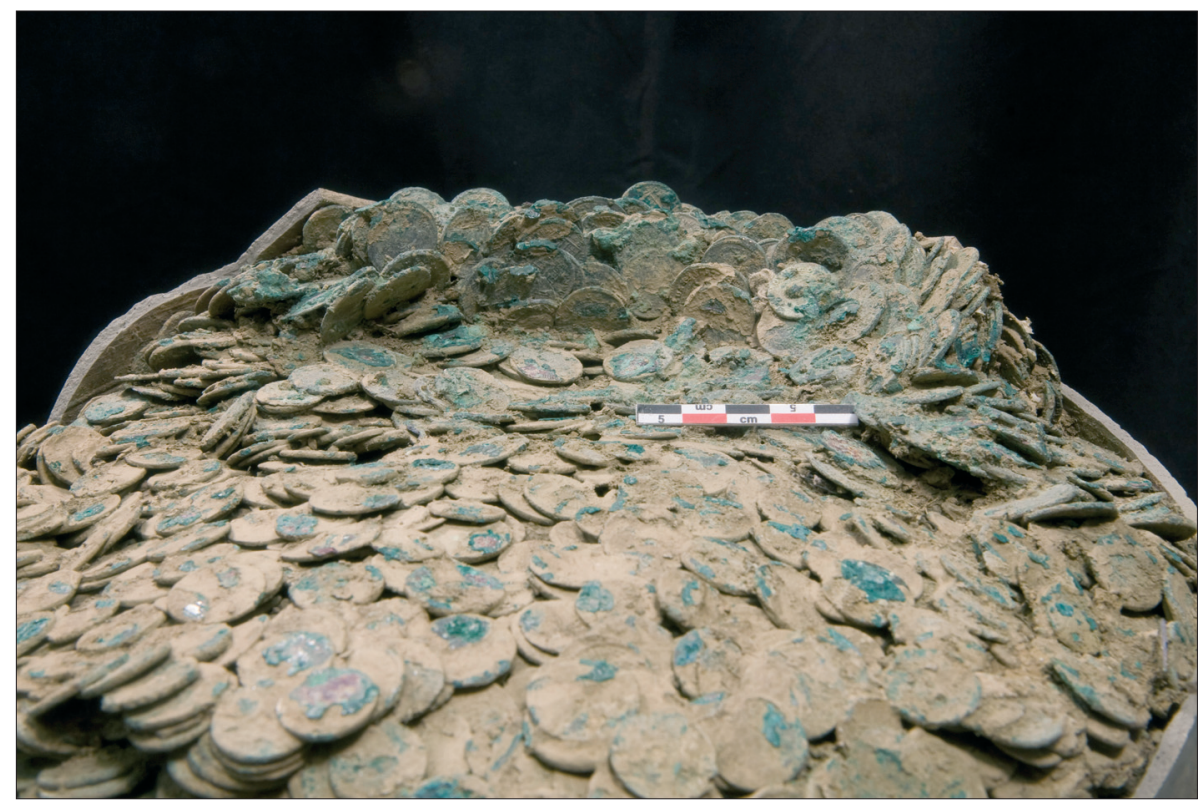

Fig. 4. 
de l'intérieur du pot cohabitaient avec des séries de monnaies inclinées sur la tranche, et souvent très concrétionnées, constituant des ensembles de tailles et de formes variables, aux contours nets et arrondis, séparés des lots voisins par de fines couches de sédiment (Fig. 4). Ces groupes de monnaies particuliers ont été interprétés comme le contenu de sacs. Leur forme ramassée et la position verticale des pièces résultent en effet de l'espace contraint dans lequel ces dernières ont été enserrées (Fig. 5). Au fil des siècles, les sacs ou les bourses (en tissu, cuir, peau, papyrus ou même écorce) se sont décomposés, laissant les monnaies qu'ils contenaient figées dans la position qu'elles occupaient. Ces enveloppes en matériaux périssables ont parfois laissé leur trace en négatif: le sac « Fait 100-101 », par exemple, était séparé des ensembles voisins par une étroite tranchée remplie d'un sédiment

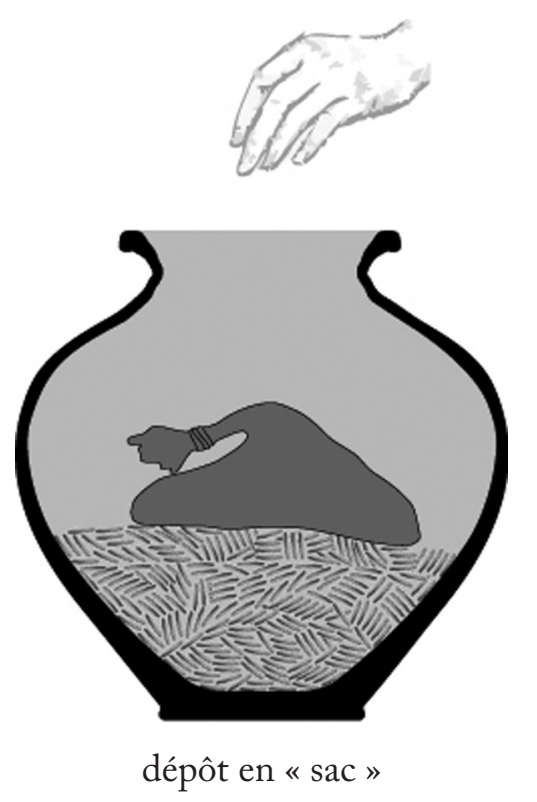

Fig. 5. - Saint-Germain-deVarreville (Manche) (Infographie : Éric Broine/CRAHAM).

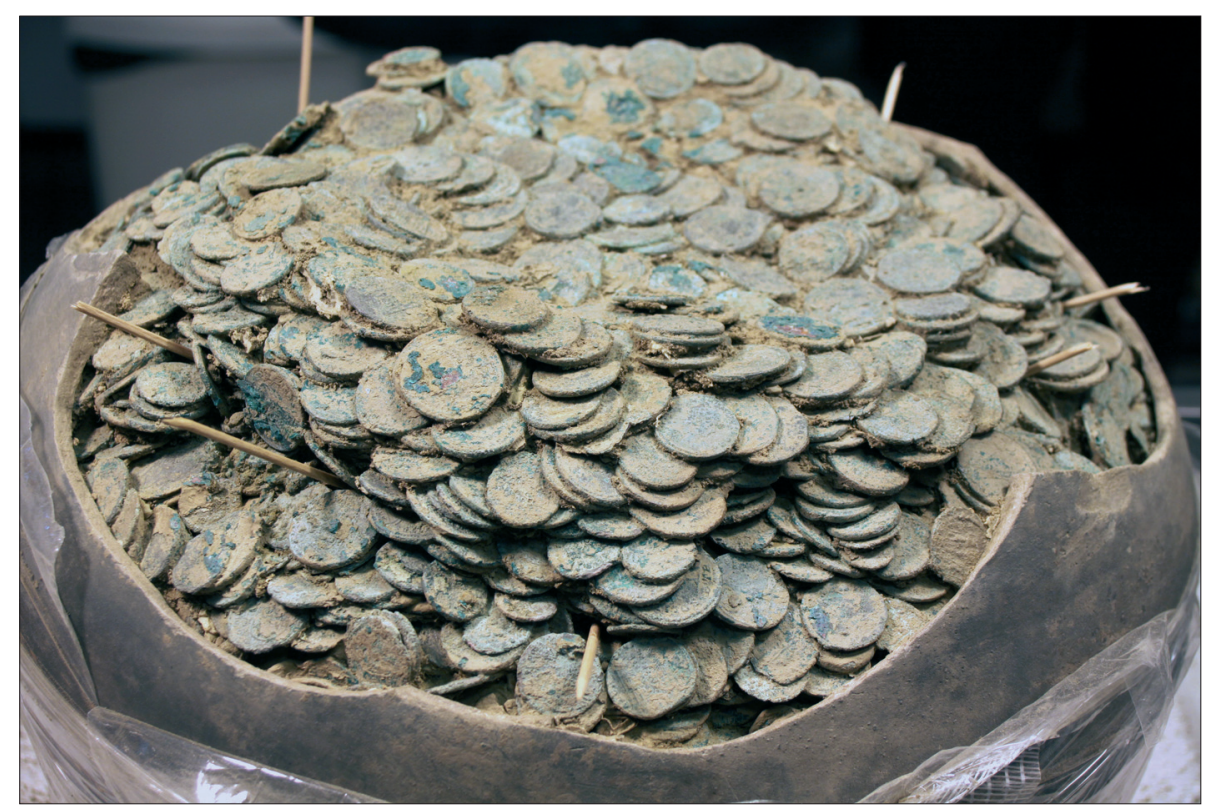

Fig. 6. 


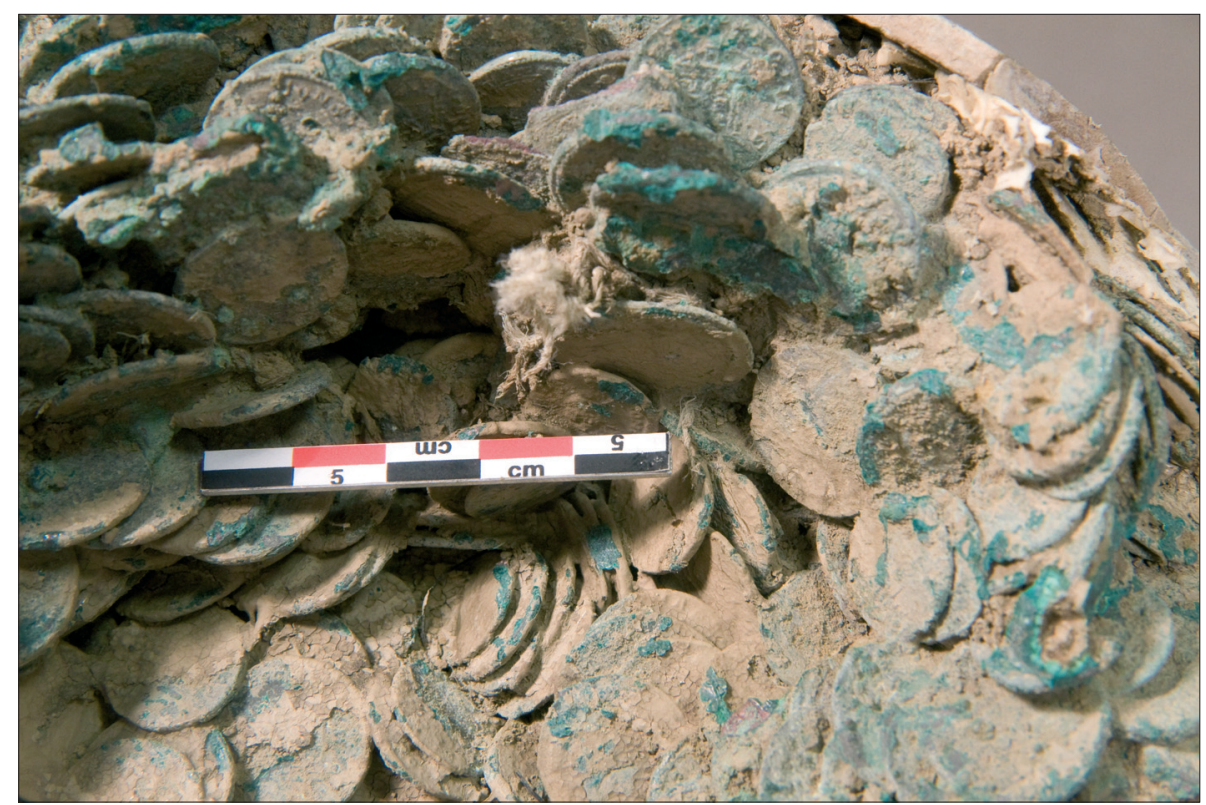

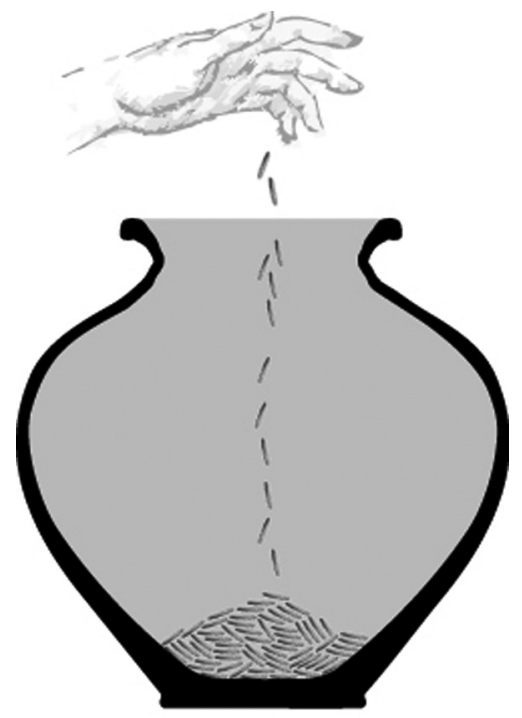

dépôt en « vrac »

Fig. 8. - Saint-Germain-deVarreville (50). Infographie : Éric Broine/CRAHAM.

Fig. 7.

argileux très fin, qui est en fait venu combler l'espace laissé vide par la disparition du sac (Fig. 6). L'existence de ces contenants est enfin soutenue par la découverte dans l'un de ces «négatifs » (Fait 104) d'une fine cordelette blanche, longue d'environ $25 \mathrm{~cm}$, enroulée sur elle-même en plusieurs boucles, et interprétée comme les restes d'un lacet ayant pu servir à nouer la poche renfermant les monnaies de ce lot (Fig. 7).

Les autres ensembles monétaires mis en évidence dans le vase étaient donc dépourvus d'autres contenants que le pot lui-même ${ }^{12}$. Les pièces y ont été versées « en pluie » par le goulot, formant des tas homogènes au sommet aplani ou légèrement pyramidal (Fig. 8). Nous avons appelé cette autre disposition des « vracs».

12 Nous n'avons pas observé de rouleaux de monnaies, bien que ce type de conditionnement ait été déjà identifié dans des trésors du premier tiers du IV siècle. Il s'agit de ceux de Cologne (Allemagne), Wettolsheim (Haut-Rhin), Fyfield (Angleterre) et surtout Seltz (Bas-Rhin). J. JAHN, "Folles in Rollen ", Jabrbuch für Numismatik und Geldgeschichte, 28/9, 1978-1979, p. 21-26. 


\section{LES RÉSULTATS DE LA FOUILLE}

La fouille du dépôt monétaire a été menée sur une quinzaine de jours au cours du mois de juillet 2012. Grâce aux moyens mis en œuvre et au soutien du Service régional de l'Archéologie, elle a permis de retracer avec précision les grandes étapes du remplissage. Elle a été également l'occasion d'apporter des informations préliminaires sur le contexte numismatique.

\section{Démontage et stratigraphie : dynamique de constitution du trésor}

Pour restituer le contenu du trésor nous avons caractérisé séparément ses différentes composantes, puis nous les avons reliées entre elles. La description des groupes de monnaies identifiés est résumée dans le tableau présenté ci-dessous (Fig. 10), tandis qu'une reconstitution (en deux dimensions) du remplissage est proposée sous forme de dessin (Fig. 9). Il apparaît ainsi que le remplissage du récipient se compose de huit phases de déposes successives, numérotées de la plus ancienne à la plus récente : trois correspondent à des sacs ; cinq à des dépôts en vrac.
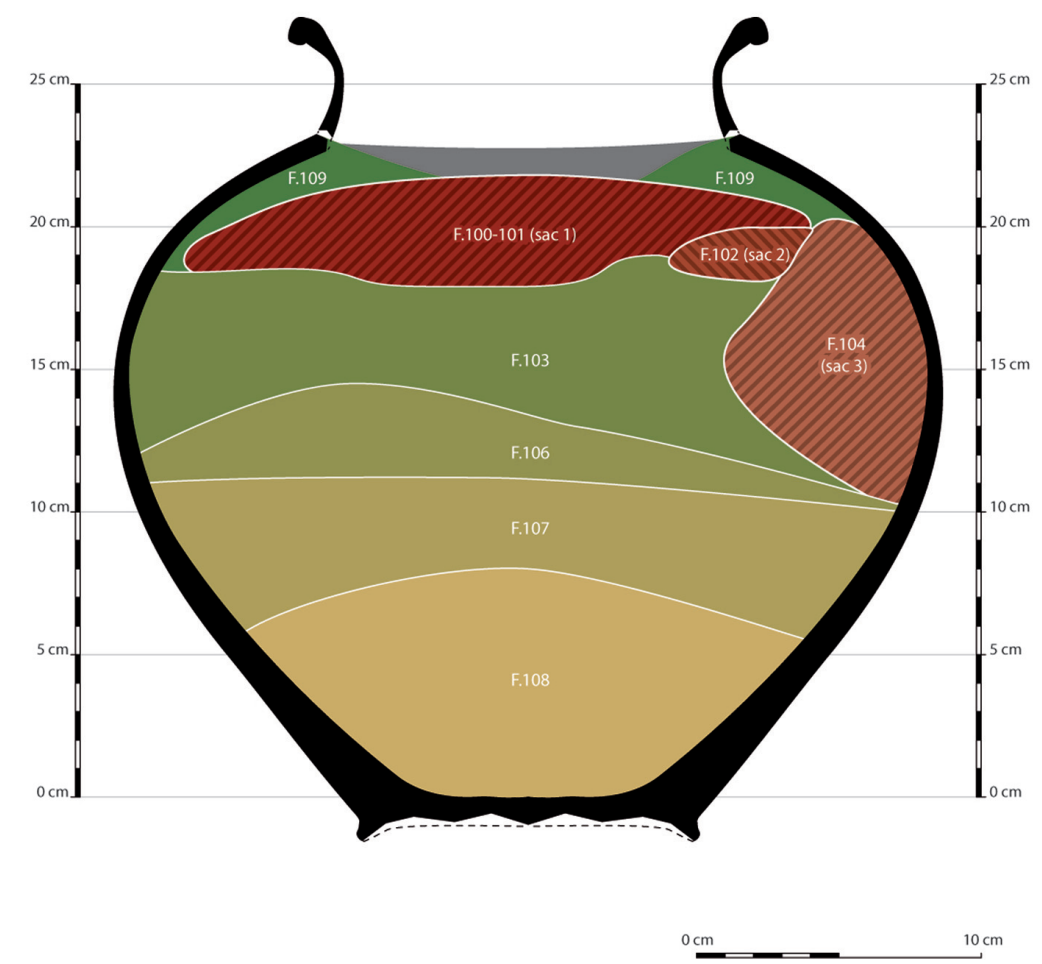

Fig. 9. 


\begin{tabular}{|c|c|c|c|}
\hline 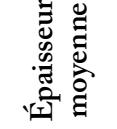 & 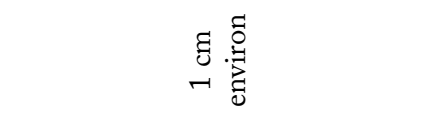 & $\begin{array}{l}\text { घี } \\
\text { m }\end{array}$ & $\begin{array}{l}\text { gี } \\
+\end{array}$ \\
\hline 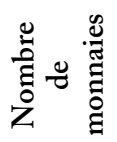 & 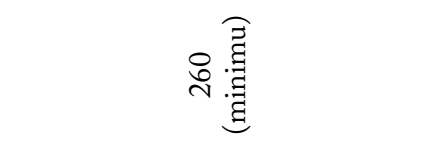 & $\stackrel{ \pm}{\sim}$ & $\begin{array}{l}\stackrel{2}{\infty} \\
\stackrel{-}{\rightarrow} \\
\end{array}$ \\
\hline 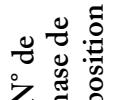 & $\infty$ & \multicolumn{2}{|r|}{$N$} \\
\hline 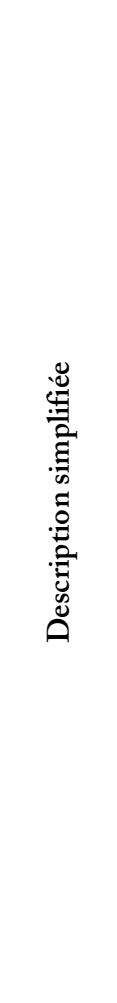 & 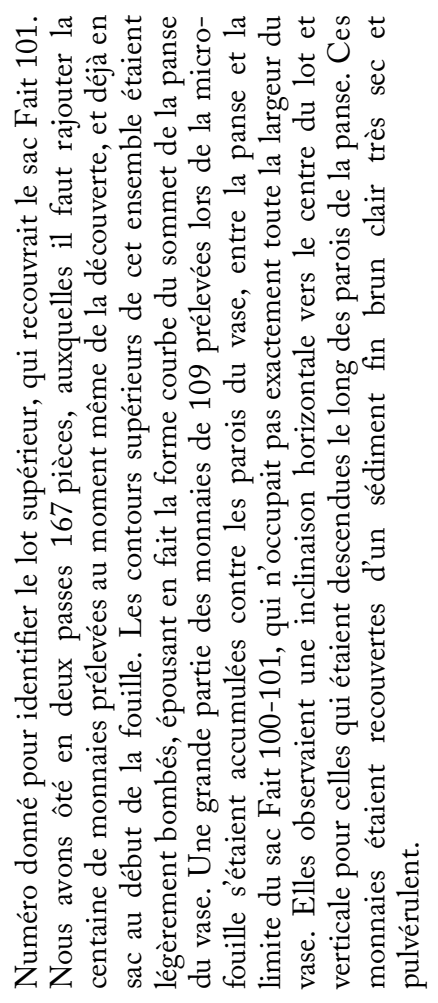 & 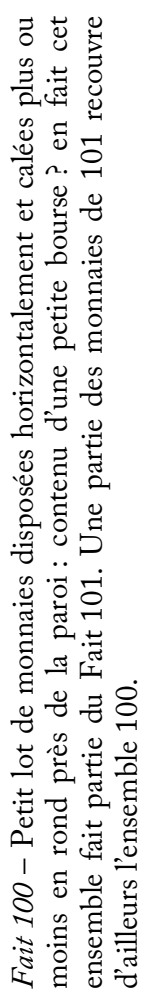 & 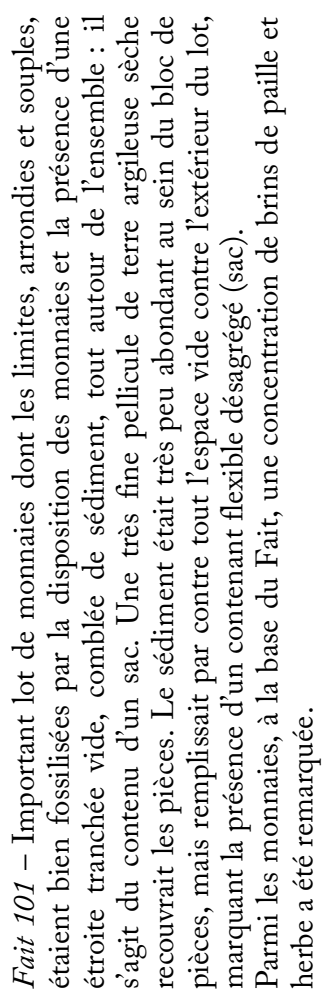 \\
\hline 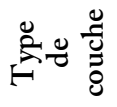 & $\stackrel{\mathscr{g}}{5}$ & \multicolumn{2}{|r|}{$\underset{\mathscr{W}}{\mathscr{E}}$} \\
\hline م. & 定 & \multicolumn{2}{|r|}{ 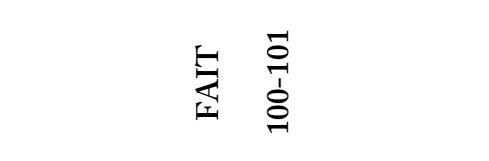 } \\
\hline
\end{tabular}




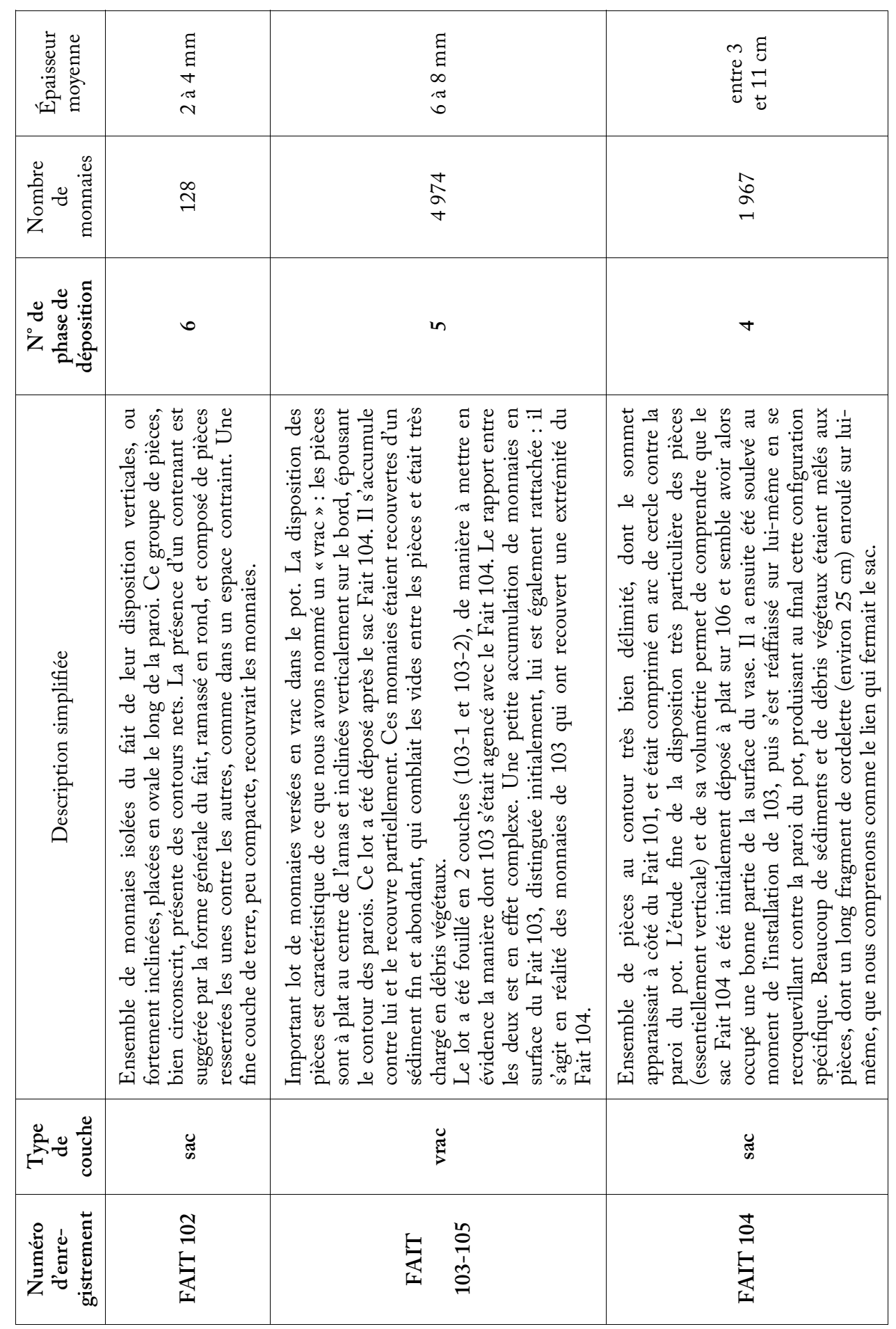




\begin{tabular}{|c|c|c|c|}
\hline 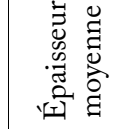 & 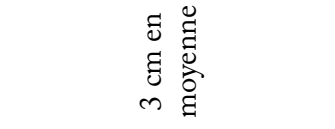 & $\begin{array}{l}\tilde{\Xi} \\
\text { m }\end{array}$ & $\begin{array}{ll}\pi & \varepsilon \\
0 & 0 \\
0 & n \\
0 & 0\end{array}$ \\
\hline 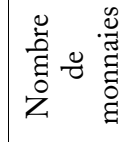 & $\vec{\curvearrowright}$ & तु & $\begin{array}{l}\text { ⿵ु } \\
\text { n }\end{array}$ \\
\hline 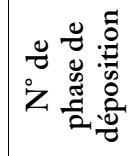 & $m$ & $\sim$ & - \\
\hline 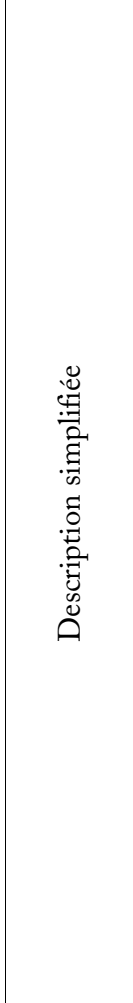 & 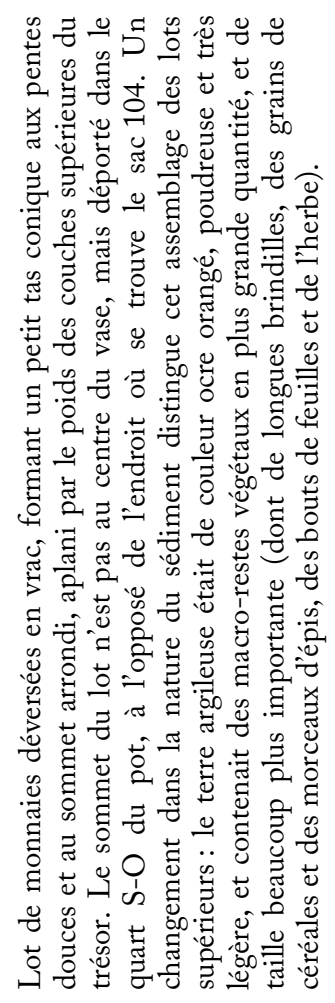 & 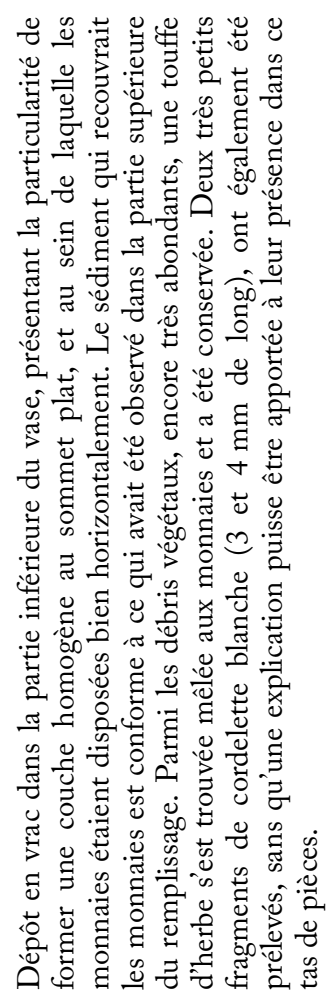 & 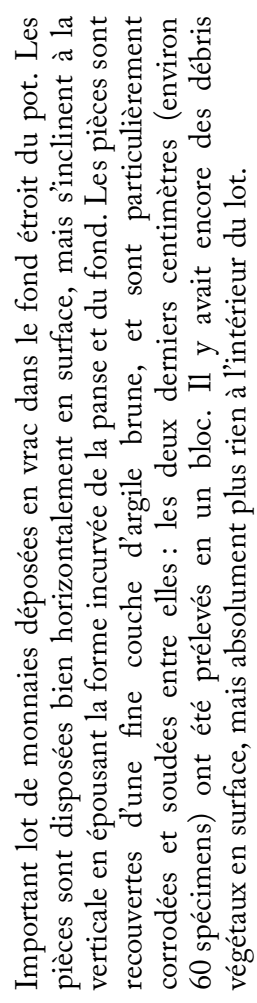 \\
\hline 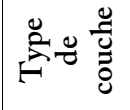 & $\ddot{\Xi}$ & $\ddot{\mathscr{J}}$ & $\stackrel{\mathscr{H}}{5}$ \\
\hline 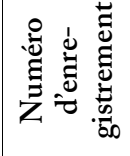 & 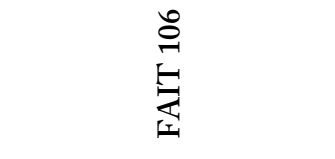 & 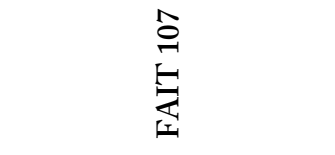 & 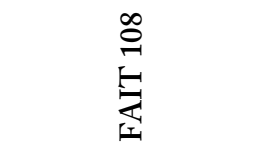 \\
\hline
\end{tabular}


Au premier abord, l'aspect compact du contenu pourrait laisser penser que le remplissage a été effectué en une seule fois. Cette lecture hâtive invite d'emblée à un examen approfondi. Attachons-nous pour cela à la moitié inférieure du récipient (Fig. 9). La caractéristique essentielle du remplissage est d'y être constituée de plusieurs couches de monnaies (Faits 106, 107 et 108) déversées en vrac et venues se loger le long de la paroi. Engendrée par un versement aléatoire « en pluie ", cette disposition découle probablement d'une volonté d'optimiser au maximum le volume disponible en comblant tous les espaces. La disparition de certains résidus organiques, notamment entre les Faits 107 et 108, cumulée à des changements de sédimentation, nous encourage à admettre que les différents ensembles identifiés ont pu rester à l'air libre dans le vase un certain temps avant d'être recouverts par un nouveau tas de pièces.

La partie supérieure du dépôt présente un scénario de dépose tout à fait différent (Fig. 9). L'agencement des monnaies y est beaucoup plus contrasté et tend surtout à montrer l'existence de monnaies groupées dans des sacs (Fig. 5). Précisément, trois groupes particuliers de monnaies ont pu être identifiés. Entre eux s'intercalent des couches de vrac. Ainsi, il apparaît que les espaces générés par la présence de sacs, ou tout simplement par la topographie du dépôt, ont été comblés par l'apport pêle-mêle de monnaies faciles à loger dans le moindre espace vide. Le plan relevé de ces lots à la fouille permet également de restituer leur position, et d'apporter, par là même, des informations sur les dynamiques de constitution du dépôt. Contre la paroi viennent se caler deux sacs de monnaies. Le premier (Fait 102) est suggéré par la disposition des pièces entre elles, inclinées sur la tranche et très resserrées les unes contre les autres. Au nord, se développe un second sac (Fait 104), qui, en adoptant la forme d'un croissant allongé et oblong, suit parfaitement la paroi nord-est du récipient (Fig. 4 et 7). Cette position très contraignante répond, selon toute vraisemblance, à une manipulation spécifique : le sac a été repoussé sur un côté du pot, afin de gagner de la place. Par conséquent, on peut imaginer que le déplacement du sac est survenu au moment même où l'ensemble monétaire 103-105 fut versé en vrac. En épousant une forme incurvée, la surface du Fait 103-105 appelle un autre commentaire. Cette légère dépression indique assez bien qu'il y a eu un enfoncement. Probablement témoigne-t-elle d'une action volontaire de la part du thésaurisateur, de manière à faire de la place pour le dépôt du sac 100-101. Celui-ci est matérialisé par la présence de monnaies se soulevant en périphérie et créant un effet de paroi. De petites accumulations linéaires de sédiment à la base indiquent vraisemblablement l'existence d'un contenant disparu.

Ainsi, l'ensemble des observations effectuées nous permet de mieux comprendre les rythmes de constitution du trésor et d'envisager un remplissage complexe, qui n'a probablement pas été réalisé en une fois. 


\section{L'environnement de la cachette du trésor : premières observations}

Plusieurs questions précises concernant l'environnement archéologique sont par ailleurs ressorties de la fouille du trésor. Notre attention a ainsi été attirée par la présence entre les monnaies, au sein de tous les ensembles individualisés, d'une terre argileuse, très fine et craquelée, séparant parfois nettement les lots. Loin d'être anodine, l'étude de ces sédiments nous semble un élément clef pour identifier le nombre de phases de déposition dans le pot, pour restituer la dynamique générale de constitution du trésor, et réfléchir sur la durée pendant laquelle le vase a pu servir de tirelire et les conditions de son développement. Les observations effectuées sur ces formations argileuses intègrent en outre celles réalisées sur les nombreux macro-restes végétaux qu'elles contenaient ${ }^{13}$.

L'abondance de ces accumulations sédimentaires et végétales pose avant tout la question de la provenance de ces éléments. Nous proposons d'expliquer les processus d'infiltration des sédiments dans le pot de la manière suivante : les très fines particules d'argile recouvrant la surface de certains ensembles monétaires, comme dans le cas du Fait 100-101, ont pu se déposer dans la cache du trésor, puis dans le pot, à la faveur d'une longue période durant laquelle la cachette n'a pas été ouverte. Ce type de formation indiquerait alors que la surface des lots est restée suffisamment longtemps à l'air libre à l'intérieur du vase pour que ces particules se soient infiltrées dans la cache, et aient recouvert, à l'instar d'un voile de poussière, le dessus du tas de pièces, avant de progressivement s'immiscer dans les interstices entre les pièces jusqu'au cœur du lot. Cette remarque est valable pour tous les dépôts de monnaies en vrac, dont les couches les plus superficielles apparaissaient toujours nappées de ces argiles de décantation craquelées. Il reste toutefois encore à comprendre comment des éléments aussi grossiers que des touffes d'herbe ou des brindilles dépassant dix centimètres de longueur se sont trouvés mêlés aux monnaies. À titre d'hypothèse, nous proposons que des débris accumulés sur le dessus de la trappe ont pu tomber à l'intérieur du vase durant la mise en place d'un nouveau lot. Il est même possible d'imaginer que le thésaurisateur a d'abord compté ses monnaies dans l'herbe avant de les descendre par poignées dans le vase, arrachant au passage la végétation et les débris sur lesquels les pièces avaient été posées. La présence d'une couche de sédiments fins recouvrant la surface des Faits 101, 103, 106, 107 et 108 a été considérée comme l'un des indices permettant de distinguer les différents ensembles monétaires entre eux. Cette caractéristique a été particulièrement importante pour différencier les trois premiers lots (108, 107 et 106), qui avaient été disposés en vrac dans la moitié inférieure du pot. Les pellicules argileuses qui les recouvraient témoignent aussi du fait que chacune de ces sommes de monnaies a été rajoutée à la suite d'une réouverture de la cachette, après une période de temps de durée indéterminée.

13 Parmi lesquels diverses sortes de graines, des brins d'herbe, des brindilles, tiges de feuilles ou très petits morceaux d'écorce, des bouts de feuilles de fougères, et parmi les macro-restes non végétaux une dent de chat et une coquille d'escargot. 
L'étude de la provenance de ces éléments non monétaires dans le vase permet en somme de reconsidérer le trésor non plus comme un seul sujet d'analyse numismatique, mais bien comme un objet archéologique, qu'il convient donc de replacer dans un contexte de découverte élargi à l'extérieur du pot. C'est ainsi que l'on peut s'interroger d'abord, à très petite échelle, sur la forme de la cache dans laquelle le vase a été déposé. Compte tenu du volume, du poids et de la forme de la céramique (pied abîmé, et dépourvu d'anse) il faut d'abord noter que ce pot ne pouvait pas être déplacé. Dans la mesure où il a été rempli progressivement, cela signifie que l'ouverture du vase était d'une façon ou d'une autre accessible depuis la surface. Nous formulons ainsi l'hypothèse que le pot avait été installé dans une petite fosse, pas nécessairement cuvelée, mais pourvue d'une trappe d'accès ou du moins d'une sorte de planche amovible qui devait permettre l'ouverture et la fermeture de la cachette, et qui devait elle-même demeurer invisible depuis la surface.

En élargissant le champ d'observation, on se demandera ensuite où pouvait être installée cette cachette dans l'environnement familier du thésaurisateur. Était-ce proche de sa maison? Proche d'une place d'échange et de commerce, sur laquelle ces monnaies pouvaient transiter ? Le long d'un chemin? Aucun site antique n'est référencé dans les environs immédiats du point de découverte, les vestiges les plus proches recensés à l'heure actuelle étant situés à deux kilomètres environ de la parcelle. Toutefois, la présence de cet ensemble monétaire, qui a été constitué sur plusieurs années, dans un contenant qui n’a pas pu être déplacé une fois rempli, montre que la cache avait été aménagée pour être rouverte à loisir, au gré des dépôts ou des ponctions. Cette accessibilité, et le fait que le trésor s'apparente davantage aux économies d'un individu qu'à un acte d'enfouissement précipité, suggèrent que la zone d'habitation ne devait pas être très éloignée de la cachette aménagée pour abriter le pécule du propriétaire des lieux.

Une dernière question peut enfin être posée : quels pouvaient être dans le paysage les marqueurs visuels qui lui permettaient de retrouver l'endroit exact où sa cagnotte était enterrée ? Sur ce point, l'identification des éléments végétaux mêlés aux monnaies permet de donner une première image du couvert végétal aux alentours directs de la cachette du vase. C'est ainsi que nous pouvons restituer un environnement plutôt ouvert, comme le prouve la présence de touffes d'herbe et de feuilles de fougères ${ }^{14}$. Le son et les balles de céréales, mis en évidence en quantité non négligeable dans certains ensembles (notamment dans le Fait 106), ne peuvent être considérés comme des indices réels de la proximité directe de champs cultivés, dans la mesure où les grains et épis

14 Il est certain que la connaissance de l'environnement végétal ne pourrait être affiné qu'en procédant à des études paléoenvironnementales (palynologie, carpologie). Lors de la fouille du pot, le prélèvement des macro-restes a été fait de manière systématique, mais aucune analyse n'a encore été réalisée. La palynologie ne nous apparaît pas comme une source documentaire plus significative que la carpologie dans le cas présent, car la quantité de sédiments accumulée dans le pot n'est pas suffisante pour livrer un échantillon de pollens statistiquement représentatif de la composition du couvert végétal autour du site de découverte. 
semblent avoir été volontairement rajoutés aux pièces, comme cela s'est vu dans d'autres trésors ${ }^{15}$, vraisemblablement dans l'idée d'absorber l'humidité retenue dans le contenant et de ralentir la corrosion des monnaies.

L'ensemble des éléments concernant les formes de la stratigraphie montre bien que le trésor de Saint-Germain-de-Varreville a été constitué en plusieurs phases. L'étude de la disposition des monnaies couplée aux observations effectuées sur les formations sédimentaires et les débris végétaux révèlent que le remplissage a été progressif et a fait l'objet de plusieurs manipulations au fil du temps. L'étude archéologique ainsi opérée du contenu permet de voir que le vase a servi en quelque sorte de tirelire dans laquelle on place, mais aussi on déplace des sacs ou des monnaies pour permettre l'insertion de nouveaux ensembles. La découverte bas-normande se différencie en cela d'autres trésors, comme celui de Villette-d'Anthon (Isère), dont l'homogénéité de la stratigraphie ont permis à $\mathrm{M}$. Amandry et à $\mathrm{S}$. Estiot de démontrer que « le pécule fut compté avant d'être introduit, en une seule fois, dans l'amphore, puis enfoui $»^{16}$. Elle constitue avant tout une réserve que l'on utilise, ce qui ne confère que plus d'intérêt à l'analyse et à la confrontation des différentes séries monétaires que renferme le dépôt pour une juste appréciation des composantes du circuit monétaire local.

\section{Premières observations numismatiques : composition et terminus}

La fouille du trésor ne fut pas une fin en soi. Elle a permis de recueillir des informations sur l'importance numérique du trésor et d'en savoir un peu plus sur sa composition.

Au fur et à mesure du démontage, nous avons procédé à un recensement systématique pièce à pièce. Au total, le nombre de monnaies inventoriées s'élève à 14528 exemplaires. Parallèlement, le comptage a été l'occasion de caractériser la composition générale du trésor, en attendant son étude exhaustive qui débutera au printemps $2013^{17}$. Ainsi, il apparaît que les pièces ont été émises sur une période qui va de ca. 310 à 348 . Ce sont exclusivement des bronzes composés de cuivre plus ou moins argenté selon l'époque concernée, que l'on appelle aussi «nummi ». La trouvaille se clôt donc à la veille de la réforme des empereurs Constance II et Constant de l'année 348, avant l'introduction d'une nouvelle dénomination, la maiorina. Par sa masse, Saint-Germain-de-

15 Ainsi dans le trésor de Neftenbach (Suisse) : H.-M. Von Kaenel et alii-Der Münzhort..., op. cit.

16 M. Amandry et alii, "Le trésor... ", op. cit., p. 118.

17 Étude qui sera précédée par le nettoyage intégral des monnaies. Cette opération sera effectuée par Guillaume Blanchet, Master 2, Université de Caen Basse-Normandie. 
Varreville se situe d'emblée parmi les plus importants trésors contemporains. Le précieux recensement des ensembles monétaires de bronzes des années 313348 publié par J.-P. Callu permet de le constater ${ }^{18}$ (Fig. 11).

Fig. 11 - Dépôts monétaires à terminus ca. 341-348.

\begin{tabular}{|c|c|c|c|}
\hline & Dépôts monétaires & $\begin{array}{c}\text { Terminus } \\
\text { enfouissement } \\
\text { (ca.) }\end{array}$ & $\begin{array}{c}\text { Total } \\
\text { monnaies }\end{array}$ \\
\hline \multirow{12}{*}{$\begin{array}{l}\text { Grande } \\
\text { Bretagne }\end{array}$} & 1. Freston (Suffolk) & $346-348$ & 3118 \\
\hline & 2. Silchester VI (Hants) & $346-348$ & 87 \\
\hline & 3. Womersley (Yorks) & $346-348$ & 3286 \\
\hline & 4. Chorleywood (Hertfords) & 348 & 4358 \\
\hline & 5. Wakefield (Yorks) & 348 & 3240 \\
\hline & 6. Woodbridge (Suffolk) & 341 & $\mathrm{NC}$ \\
\hline & 7. Woolaston & $341-348$ & 183 \\
\hline & 8. Langwith & $341-348$ & 1451 \\
\hline & 9. Llanbethery & $341-348$ & 739 \\
\hline & 10. Bryndedwydd & $341-348$ & 115 \\
\hline & 11. Bishops Wood & $341-348$ & 16469 \\
\hline & 12. Maiden Castle & $341-348$ & 7 \\
\hline Bénélux & 13. Tournai IV & $341-346$ & 13 \\
\hline \multirow{7}{*}{ France } & 14. Saint-Germain-de-Varreville (Manche) & $341-348$ & 14435 \\
\hline & 15. La Chapelle-lès-Luxeuil (Haute-Saône) & 342 & 15518 \\
\hline & 16. Cahors-Saint-Georges (Lot) & 345 & 236 \\
\hline & 17. Les Granges-Gontardes (Drôme) & 348 & 27 \\
\hline & 18. Frontignan I (Hérault) & 348 & 18 \\
\hline & 19. Frontignan II (Hérault) & 348 & 66 \\
\hline & 20. Poitiers (Vienne) & 348 & 63 \\
\hline
\end{tabular}

18 J.-P. CALlu, Inventaire des trésors de bronze constantiniens (313-348), Wetteren, 1981, p. 13-68. À cet inventaire, il convient d'ajouter les références suivantes : J. Kent, The Roman Imperial Coinage, vol. VIII, The family of Constantine I (A.D. 337-364), Londres, Spink, 1981, table 4, p. 84 ; A. Geissen, B. Päff gen et G. Quarg, «Die Münzen des Hortfundes aus dem Königsforst », Bonner Jahrbücher, 184, 1984, p. 401-477 ; D. Gricourt, La Chapelle-lès-Luxeuil (Haute-Saône), 15518 nummi constantiniens, Paris, Bibliothèque nationale de France, 1999 (Trésors Monétaires ; XVIII). 


\begin{tabular}{|c|c|c|c|}
\hline & Dépôts monétaires & $\begin{array}{c}\text { Terminus } \\
\text { enfouissement } \\
\text { (ca.) }\end{array}$ & $\begin{array}{c}\text { Total } \\
\text { monnaies }\end{array}$ \\
\hline \multirow{4}{*}{ Allemagne } & 21. Westick (Nordrhein-Westfalen) & 344 & 56 \\
\hline & 22. Neustadt-Weinstrasse (Rheinland-Pfalz) & $346-348$ & 46 \\
\hline & 23. Prüm (Rheinland-Pfalz) & $341-346$ & 65 \\
\hline & 24. Königsforst & $347 / 348$ & 3636 \\
\hline Autriche & 25. Bruckeudorf (Burgenland) & 348 & 234 \\
\hline \multirow{2}{*}{ Espagne } & 26. Tarragone (Tarragone) & 348 & 328 \\
\hline & 27. Barcelone (Barcelone) & 348 & 56 \\
\hline \multirow{2}{*}{$\begin{array}{l}\text { Afrique } \\
\text { du Nord }\end{array}$} & 28. Constantine (Algérie) & 348 & 255 \\
\hline & 29. El Arrouch (Algérie) & 348 & c. 800 \\
\hline Yougoslavie & 30. Dobrotine (Kossovo) & 348 & 1374 \\
\hline \multirow{3}{*}{ Grèce } & 31. Attique & $343-346$ & 139 \\
\hline & 32. Kastelliou Kisamou (Crète) & 348 & 120 \\
\hline & 33. Thasos (Macédoine) & 348 & 32 \\
\hline Turquie & 34. Région de Smyrne (Ionie) & 348 & 46 \\
\hline $\begin{array}{l}\text { Proche- } \\
\text { Orient }\end{array}$ & 35. Palestine & 343 & 525 \\
\hline Égypte & 36. Fayoum & 343 & 6141 \\
\hline
\end{tabular}

L'excellent état de conservation d'un grand nombre de monnaies a permis de cerner - sous réserve d'une étude numismatique plus détaillée - les grandes composantes du dépôt (Fig. 12). Il commande de lui-même le commentaire. Ainsi, il est apparu que le monnayage antérieur à la réforme de 318 (principalement au type Soli invicto comiti) n'est que résiduel. Les émissions des années 318-324 et 324-330, avec les types Victoriae laet princ perp, Beata tranquillitas, Sarmatia devicta, et Providentiae augg / caess, sont mieux représentées, mais restent modestes par rapport au numéraire de la période 330-341. Plus des deux tiers du trésor consistent en effet en nummi offrant le revers Gloria exercitus à une ou deux enseignes, ou l'un des types urbains qui lui sont associés, Urbs Roma et Constantinopolis. Dans cet ensemble, il semblerait que les émissions de la période 330-335 soient néanmoins plus nombreuses. À cela, s'ajoute la présence régulière d'imitations. Parmi celles-ci, les faussaires ont, semble-t-il, privilégié les reproductions de nummi aux revers Gloria exercitus (deux enseignes), Urbs Roma et Constantinopolis. Enfin, les frappes des années 341-348 au type Victoriae dd auggq nn, qui clôturent la thésaurisation, s'avèrent, quant à elles, 


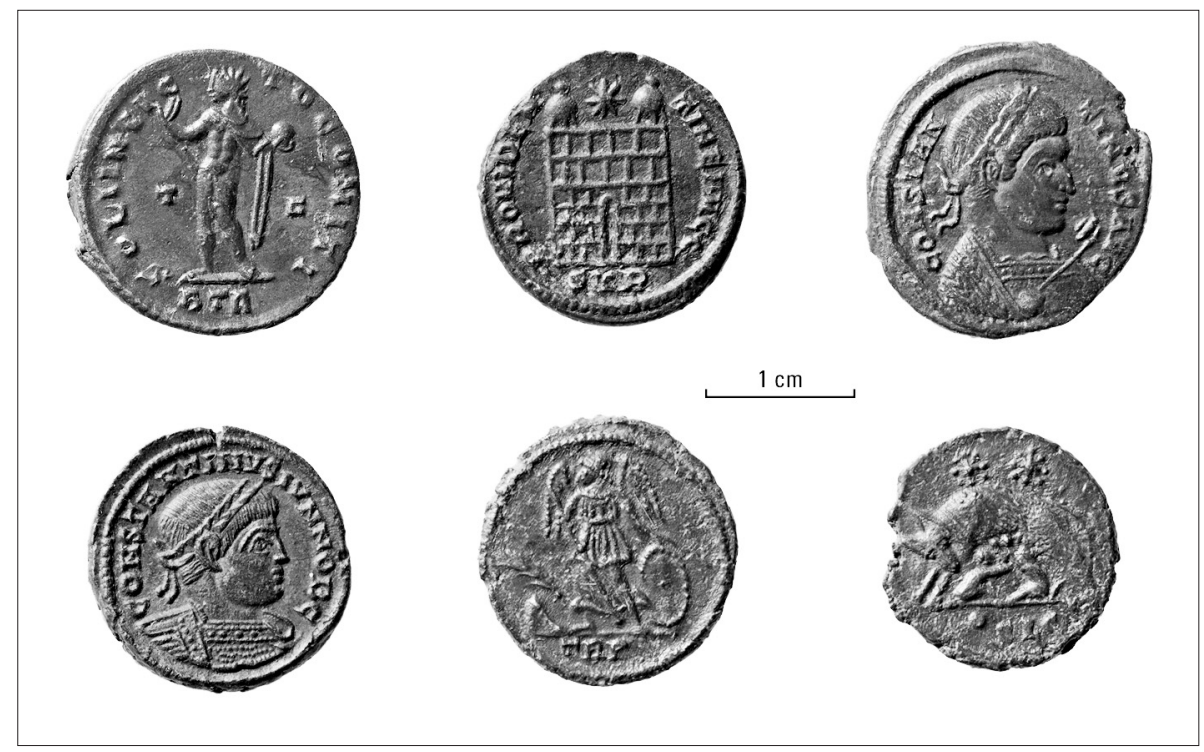

Fig. 12.

très discrètes. D'un point de vue structurel, il ne semble pas que les pièces, présentes dans les niveaux inférieurs du trésor, diffèrent de celles rencontrées dans les niveaux supérieurs. Les monnaies sont diffuses et extrêmement brassées entre elles. Une certitude s'impose donc : les différentes émissions recensées circulaient et alimentaient conjointement le circuit monétaire local. De façon complémentaire, il sera intéressant de voir s'il en est de même à l'intérieur des sacs. Y-a-t-il eu un tri sélectif du numéraire lors de leur constitution ? Seule l'étude numismatique à venir nous permettra de répondre.

Pour l'heure, tenons-nous en aux premières observations. Qu'est-ce que nous apprennent les composantes générales du trésor ? On retiendra un aspect principal : la prééminence des séries des années 330. Le constat est d'importance, car il nous amène à penser que le dépôt a été constitué sur le tard. Toutefois, il ne prend son intérêt que si on le met en relation avec un autre événement. En effet, le profil compacté de l'ensemble pourrait être une réaction aux mesures pondérales de 336, qui réduisent le nummus à l'état de piécette ${ }^{19}$. Dans ce contexte, la formation du trésor interviendrait sur une courte période, peut-être une quinzaine d'années, entre 336 et ca. 348, date de clôture du dépôt.

19 Parallèlement, la part du plomb augmente et atteint même jusqu'à $16 \%$ dans les émissions frappées à Trèves. G. Depey rot, Le numéraire gaulois du IV siècle. Aspects quantitatifs, livre i, Oxford, 1982, p. 75 (BAR International Series ; 127i). 


\section{CONCLUSION}

L'analyse du trésor de Saint-Germain-de-Varreville a permis de recueillir d'importantes informations sur sa constitution, et de démontrer une fois encore toute la pertinence d'une approche archéologique appliquée à ce type de découverte.

Les résultats enregistrés permettent d'en esquisser l'histoire à grands traits. Riche de 14528 nummi, le trésor est constitué de monnaies émises sur une quarantaine d'années, entre 310 et 348 . Comme le révèlent les premières observations numismatiques, le cœur de son contenu forme un ensemble compact de monnaies produites sur une période courte, centrée sur la décennie 330. Or, ce n'est probablement pas le hasard qui explique un tel resserrement du profil monétaire, mais bien une raison d'ordre économique, qui serait consécutive à la réduction pondérale du nummus survenue en 336. On comprend, dès lors, pourquoi le thésaurisateur s'est contenté de mettre spécifiquement à l'abri de bonnes monnaies. Toutefois, cette interprétation est incomplète. Les conclusions émises lors de la fouille permettent d'aller plus loin. Elles conduisent à reconnaître que Saint-Germain-de-Varreville ne fut pas seulement un capital d'argent immobile, introduit en une seule fois dans le vase et destiné à ne pas être dépensé. Observations stratigraphiques et agencement des monnaies indiquent un remplissage organisé et progressif au fil du temps, par ajout voire retrait graduel de monnaies. Il serait donc inexact de penser qu'il ne fut que le fruit d'une thésaurisation. Peut-être faudrait-il alors imaginer qu'il constituait le fond de roulement, l'encaisse d'une personne qui avait à utiliser au quotidien des monnaies.

L'histoire est donc celle d'un particulier accumulant des économies sur plusieurs années, mort avant d'avoir pu en profiter et sans avoir révélé l'emplacement de sa cachette. Ultime témoignage de la vie de cet individu, ce trésor nous a donné l'occasion d'entrevoir aussi un fragment de vie d'un Gallo-Romain $\mathrm{du} \mathrm{IV}^{\mathrm{e}}$ siècle après J.-C., aux activités professionnelles relativement lucratives, en train de mettre à l'abri ses économies dans une cachette, rouverte à chaque fois qu'il avait besoin soit de puiser, soit de rajouter des sommes d'argent à son pécule. 


\title{
Résumé
}

La fouille du trésor monétaire de Saint-Germain-de-Varreville (Manche) : stratigraphie d'un pécule de 14528 nummi (première moitié du IV ${ }^{\mathrm{e}}$ siècle). - Constitué de 14528 nummi s'échelonnant de $c a$. 310 à 348 de notre ère, le trésor de Saint-Germainde-Varreville (Manche) se rattache à une famille d'enfouissements clos dans le courant de la décennie 340 et précédant la réforme des empereurs Constance II et Constant de l'année 348. Exceptionnel par le nombre de monnaies comptabilisées, il l'est aussi par son état de conservation : l'ensemble des monnaies était contenu dans un gros vase en céramique commune, inviolé depuis des siècles. Ces conditions particulières ont encouragé le développement d'un projet de fouille ambitieux.

Réalisée au service de numismatique du Centre Michel de Boüard-CRAHAM, la fouille du trésor avait pour objectif d'appréhender le comportement du thésaurisateur face à son pécule. Le protocole d'étude suivi s'est attaché à reconnaître les différentes composantes du trésor et à en restituer la stratigraphie. Les résultats obtenus ont ainsi permis de révéler un remplissage organisé et progressif au fil du temps, par ajout voire retrait graduel de monnaies disposées en vrac ou dans des sacs.

Mots clefs : trésor - nummi - fouille - stratigraphie - mode de remplissage - sédiments - macro-restes - thésaurisateur - $\mathrm{sac}-\mathrm{vrac}-\mathrm{IV}^{\mathrm{e}}$ siècle.

\begin{abstract}
Archaeological results from the Saint-Germain-de-Varreville (Manche) site: the stratification of 14528 coins from the first half of the fourth century. - The treasure of Saint-Germain-de-Varreville (Manche) contains 14528 coins dating from 310-348 A.D., which were found in a closed and undisturbed ceramic container buried before 348 and the reforms of Constance II and Constant. The number and the preservation of the coins are exceptional.

The examination of this site by the numismatic service of the Centre Michel de Boüard-CRAHAM hoped to understand the owner's behaviour and discover the different types of coins and their deposit or gradual withdrawal, in bulk or in bags, over time.
\end{abstract}

Key words : treasure - coins - archaeological site by strata - savings - sediments - hoarding - bags $-4^{\text {th }}$ century A.D. 